\title{
Research on the Barrier-free Package Design Based on the Oriented Needs of the Elderly
}

\author{
Xiaohui Shen ${ }^{1, *}$ Wei Yan $^{1}$ \\ ${ }^{1}$ Art College, Northeast Electric Power University, Jilin, Jilin 132000, China \\ *Corresponding author. Email:2512317942@qq.com
}

\begin{abstract}
How to actively respond to the aging of the population and further improve people's livelihood and well-being is a practical problem that needs to be solved urgently and it is also the purpose of this research. Through the comprehensive application of observation method, case analysis method and literature research method, this article conducts a comprehensive analysis of the Chinese elderly population and conducts an in-depth exploration of the design optimization of barrier-free packaging for the oriented needs of the elderly. It is concluded that the package design should be people-oriented and pay attention to the physical and mental characteristics of the elderly, such as the decline of visual judgment, the slowness of action capability, and the pessimism of psychological emotions. The packaging should have the characteristics of information transmission, usage experience, and barrier-free emotion communication, not only to bring safety and convenience to the elderly in terms of use, but also to give the elderly sufficient humanistic care. This conclusion also has certain theoretical significance and practical value.
\end{abstract}

Keywords: The elderly, Oriented needs, Barrier-free package design.

\section{INTRODUCTION}

Barrier-free design emphasizes that in a modern society with highly developed science and technology, all public space environments as well as the planning and design of various building facilities and equipment related to human basic necessities must fully consider the usage requirements of people with different degrees of physical disability and those with reduced normal capacity for action (such as the disabled and the elderly) [1]. The barrier-free package design based on the oriented needs of the elderly aims to investigate and study the applicability and practicality of the elderly in the process of packaging and using, analyze and understand the universal and common characteristics and special individual needs of the elderly, then make the product packaging achieve zero-impediment communication and interaction with the elderly in terms of information transmission and usage experience through scientific and rigorous design, and balance the objective contradiction between the necessary life needs of the elderly and the hysteresis quality of packaging design with diligent efforts.

\section{HUMAN FACTORS ANALYSIS OF THE ELDERLY IN CHINA}

According to statistics, the current elderly population accounts for a huge proportion of China's demographic structure, the number is obviously increasing year by year, and the team of the elderly population continues to grow and develop.

\subsection{The Decline of Visual Judgment}

With the gradual decline of body functions, the eye structure of the elderly also appears to be aged and degraded, and their vision is rapidly decreasing. At the same time, the visual perception ability of the elderly for spatial orientation and visual modeling is weakened. They have obstacles of different severity when recognizing and judging objects near or far and they are prone to visual blur and ghosting when recognizing and judging objects. In addition, with the aging of the eye structure, the 
incidence of eye diseases and related diseases in the elderly has also increased. For example, cataract, glaucoma, macular degeneration, muscae volitantes, vasa sanguinea retinae blockage, trachoma, xerophthalmia, heterochromia iridis, and epiphora are common vision killers in the elderly.

\subsection{The Slowness of Action Capability}

The growth of age will not only cause the disease of the ocular system of the elderly, but also the aging of the muscle structure and tissues. For example, hyperostosis and other related structural changes will greatly affect the flexibility of the muscles of the elderly, and it is extremely easy to cause amyotrophy. The most direct impact of amyotrophy is the deterioration of the dexterity of the physical activities of the elderly, and the prolonged reaction time to external things. Under normal circumstances, the muscle strength of the elderly is only half of that of the young and middleaged. In addition to the changes in muscle structure and tissues, the elderly also have different degrees of decline in organ function, which will slow down their body reaction and their ability to move.

\subsection{The Pessimism of Psychological Emotions}

The decline of physiological function is one of the manifestations of morbidity in the elderly. In terms of psychological emotions, the elderly also show negative and pessimistic states in different situations, which is another manifestation of morbidity. Emotionally, the elderly generally have the psychological characteristics of low sense of security, sometimes they may have extreme emotions, and their ability to adapt to the outside world deteriorates, showing psychological and emotional inferiority, loneliness, and helplessness. In addition, due to the restriction of physiological conditions, some elderly people also reject external things and greatly resist new things. They take longer to receive new things, their ability to adapt to the new environment is weakened, and they lack spiritual and emotional comfort and sustenance.

\section{OPTIMIZATION OF BARRIER- FREE PACKAGE DESIGN FOR THE ORIENTED NEEDS OF THE ELDERLY}

Regarding the package design itself, Japanese scholar Taku Ibuki once proposed the principle of "markedness, understanding, and favorable impression" [2]. In order to achieve the purpose of people-oriented service, the barrier-free package design based on the oriented needs of the elderly should also follow the corresponding design principles to optimize the barrier-free design. The package design optimization is specifically manifested in aspects such as the visual information element transmission of the packaging, the usage experience of the packaging, and the emotional communication and so on.

\subsection{Design Optimization of "Barrier-free Information Transmission"}

In order to make the barrier-free package design more recognizable for the oriented needs of the elderly, the graphic design part, the text and layout design part and the color design part in the barrierfree package design should be distinct, clear and complementary, so as to jointly achieve the humanized purpose of barrier-free package design.

\subsubsection{Concise Graphic Symbols}

The graphic symbols in the package design include elements such as the brand logo, the specific image of the product, the abstract image after the font change, and other auxiliary decorative images. Psychologists have learned that simple, concise and complete graphics such as rectangles, circles, and straight lines meet the requirements of human visual perception better. In barrier-free package design, for the elderly with memory impairment, simple and pure graphic symbols are easier to identify and remember. In the package design, the graphic symbols of the main image should be highlighted so that the graphic symbols can directly and fully display the product. In addition to the main image, package design must also follow the principle of unifying form and content. Therefore, package design can also be combined with the use of oriented symbols. The oriented graphic symbols have clear indication, and they are the most concise and eye-catching, providing the most efficient visual guidance for the elderly.

\subsubsection{Clear Text Layout}

In package design, text symbols are the most direct and effective way to spread product information content with the general public. The text in the packaging is mainly divided into three parts. The first is the basic text. The basic text is an introduction to the basic product information such 
as company name and product name, and its design should be standardized and rigorous. The second is the explanatory text. The explanatory text includes an introduction to the product's performance, ingredients, date, place of origin, and matters need attention. There are many texts in this part, therefore, when designing, attention should be paid to the readability of the text content. It is recommended to use standard text in Chinese and sans-serif font in English. In the layout design of the text, it's needed to follow the general law of reading from left to right and from top to bottom, and pay attention to the distance between words, lines, and groups. The third is the advertising text. The advertising text is an unnecessary part of the content in the package design, intending to promote the product. When designing, this part should be vivid and refined, highlighting the main text information and weakening the secondary text information. In addition, when arranging text, elements such as lines can be appropriately added for visual guidance, providing visual guidance for information reading for the elderly.

\subsubsection{Steady Color Performance}

American psychologist and art theorist Rudolf Arnheim pointed out in "Visual Psychology": Color can express emotion, which is an irrefutable fact [4]. Vision is the most important existence in the human sensory system. The vision of the elderly has a prerequisite for the perception of color, so color has a better guiding effect for the elderly [5]. In the color design of barrier-free packaging, the following aspects should be paid attention to. The first is the eye-catching nature of colors. Color is the design element that most directly attracts the attention of the audience. For important information content, it can be guided by obvious color blocks. At the same time, the color resolution should be strong, try to reduce the design and use of similar colors in the chromatic circle, and try to choose the 180-degree relative color to enhance the recognition. For example, the combination of black and white has strong contrast, simplicity and tension, which makes people feel clear. Conversely, the matching applications between green and blue, red and purple, and gray are often not easy to distinguish for the elderly with visual impairment. The second is the recognition of colors, that is, according to the VI visual identification system of the product, the brand image is highlighted through standard colors. The third is the symbolism of colors. Different color systems represent different character languages. The fourth is the emotional nature of colors. The understanding and preference of color will vary according to the corresponding age group, which is unblamable. For example, older people prefer stable colors such as blue, gray, and brown.

\subsection{Design Optimization of "Barrier-free Usage Experience"}

The "barrier-free usage experience" in the barrier-free package design means that the packaging can meet the convenience, safety, and comfort needs of elderly consumer groups when the product is used, realizing the accessibility in the use process such as packaging opening and closing.

\subsubsection{Safe and Suitable Packaging Materials}

At present, there are an unusually wide variety of packaging materials in the packaging market and they are showing a diversified development trend. There are natural packaging materials, artificial packaging materials and multi-layer packaging materials, and there are both single packaging materials and synthetic packaging materials. When choosing materials for barrier-free packaging, attention should be paid to the economic applicability of packaging materials. According to the physiological characteristic of the weakened muscle strength of the elderly, it's recommended that the selected packaging materials are light in weight. For example, paper packaging materials in natural materials are typically characterized by light weight, strong flexibility, and easy folding operation. In addition, the use of new functional materials provides a great safety guarantee for barrier-free packaging. For example, these functional materials, such as time-temperaturesensitive indicating materials, gas-sensitive indicating materials, photovoltaic smart indicating materials, and bio-microbial sensing smart packaging materials, will make corresponding scientific judgment and reaction based on changes in different environments. Through the use of new packaging materials, the specific purpose of packaging can be realized, and technical assistance can also be provided for the usage experience of barrier-free packaging for the elderly.

\subsubsection{Scientific and Rigorous Packaging Structure}

When designing the structure of barrier-free packaging, the physical characteristics of the 
packaging materials and the action capability characteristics of the elderly should be fully combined, and the convenience and safety of the packaging structure should be fully considered, combining the relevant principles of human factors engineering to scientifically and rigorously design the packaging structure. For example, it's necessary to calculate the grip size of the elderly and design the opening of the packaging strictly according to the data size, so as to make it convenient for the elderly to use the packaging when opening and closing. In addition to rigorous size design, further in-depth research on packaging can be combined with physics principles. For example, when designing bottle-shaped packaging, one can design the texture of the bottle mouth and the sliding cap to increase the friction at the bottle mouth and the sliding cap, which not only facilitates the opening and closing of the packaging, but also prevents the packaging from slipping off the hand. For packaging with high safety index requirements such as medicines, structural sub-packaging design can be carried out, and through the rigorous packaging structure design, the elderly can be provided with the safety guarantee of packaging usage. Wright said that "form and function are one." [6]. Therefore, in addition to realizing the functionality of barrier-free packaging structure, it is also necessary to enhance the sense of form and beauty of the packaging.

\subsection{Design Optimization of "Barrier-free Emotion Communication"}

When designing barrier-free packaging for the elderly, in addition to paying attention to the visual and physiological characteristics and behavioral characteristics of the elderly, it's also necessary to accurately grasp the psychological characteristics and special aesthetic tastes of the elderly. Psychological emotions are also a major obstacle faced by the elderly. The barrier-free emotion communication is mainly expressed through visual information

\subsubsection{Giving Psychological Care to the Elderly}

The application of different styles of graphic symbols and colors in package design will bring different visual perception and psychological feelings to the elderly. In the barrier-free package design, the visual performance of the picture is carried out through visual symbols such as graphics, texts and colors that fit the psychology of the elderly. This, to a certain extent, can increase the sense of security of the elderly, relieve their negative psychological emotions, and give the elderly a sense of comfort and sufficient humanistic care on the psychological level.

\subsubsection{Being in Line with the Aesthetic Taste of the Elderly}

In the transition from mainstream society to marginal roles, some elderly groups have also diminished their curiosity and attention to external things, easily rejecting new things and tending to have objection psychology against external activities. At the same time, the elderly have their unique personalities, preferences and taboos, and they tend to show great resistance and rejection to phenomena that do not fit their own judgment and cognition. In severe cases, they may even lose control of their emotions. When designing barrierfree packaging, designers should empathize and understand the mood and preferences of the elderly, and express their true feelings of care and respect for the elderly through graphics and texts.

\section{CONCLUSION}

Through a comprehensive understanding of the physical and mental characteristics and behavioral abilities of the elderly in China, as well as a systematic analysis of the oriented needs of the elderly, this research proposes some specific optimization methods for barrier-free package design suitable for the oriented needs of the elderly. Through the implementation of these optimization methods, the purpose is to effectively provide safe and convenient guarantee for the packaging usage of the elderly, so that barrier-free packaging can truly provide humanized services, meet the physical and mental needs of the elderly, achieve or even exceed their expected goals, bring blessedness to the elderly and promote the harmonious development of Chinese society, which is of great practical significance. At the same time, through the optimization design research and discussion on the three aspects of "barrier-free information transmission", "barrier-free usage experience", and "barrier-free emotion communication" in barrierfree packaging design, this research hopes to provide some theoretical cornerstones for more research scholars, and at the same time, it's hoped to arouse the Chinese public's thinking about barrier-free packaging design and raise the awareness of barrier-free design, which also has 
certain theoretical significance and value significance.

\section{AUTHORS' CONTRIBUTIONS}

Xiaohui Shen is responsible for the redacting of this paper, and Wei Yan revised the paper.

\section{REFERENCES}

[1] Zhang Hongying. Analysis on the Barrier-free Design of Buildings $[\mathrm{J}]$. Technology Wind, 2012(10):196. (in Chinese)

[2] Zhang Lihong. The "Markedness, Understanding, and Favorable Impression" of Package Design $[\mathrm{J}]$. Screen Printing Industry, 2002(05):31-33. (in Chinese)

[3] Cai Yongrong. Research on Simplified Graphic Design in Contemporary Package Design [J]. Ming Ri Feng Shang, 2020(24):34. (in Chinese)

[4] Rudolf Arnheim. Visual Psychology [M]. Beijing: China Social Sciences Press, 1984. (in Chinese)

[5] Deng Feijie, Hu Xi. Research on Color Design Strategy and Application of Daily Necessities for the Elderly $[\mathrm{J}]$. Art and Design (Theory), 2019,2(03):115-117. (in Chinese)

[6] Zheng Fanglei. Exploration of the Structure and Form Design of Packaging [J]. Green Packaging, 2020(11):54-57. (in Chinese) 